\title{
Assessment of medical professionalism: preliminary results of a qualitative study
}

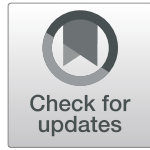

\author{
Warren Fong ${ }^{1,2,3,4^{*}} \mathbb{D}$, Yu Heng Kwan ${ }^{5}$, Sungwon Yoon ${ }^{5}$, Jie Kie Phang ${ }^{1}$, Julian Thumboo ${ }^{1,3,5}$, \\ Ying Ying Leung ${ }^{1,2,3}$ and Swee Cheng $\mathrm{Ng}^{1,2}$
}

\begin{abstract}
Background: The importance of medical professionalism has been well documented in the literature. Cultural background affects the constituents of professionalism. However, few studies have explored the domains of medical professionalism in an Asian context. We aim to describe the views and experiences of both patients and faculty on medical professionalism in an urban Asian city state.

Methods: Data for this qualitative study were collected through focus group discussions (FGDs) with patients and in-depth interviews (IDIs) with faculty members. The IDIs and FGDs were audio-recorded and transcribed verbatim. Thematic analysis was conducted by two independent coders using a priori framework derived from Professionalism Mini Evaluation Exercise (P-MEX). Sociodemographic data of faculty members and patients were obtained through brief questionnaires.

Results: A total of 31 patients ( $58.1 \%$ male, all had visited healthcare facilities within the past year) and 26 faculty members (42.3\% male, 38.5\% from medical disciplines, median years as faculty is 11$)$ participated in this study. The results supported the four domains of medical professionalism - Doctor-patient relationship skills, Reflective skills, Time management and Inter-professional relationship skills. Two new sub-domains which emerged from data were (1) communicated effectively with patient and (2) demonstrated collegiality.

Conclusions: The domains of professionalism in Singapore were similar to previous studies. This study allows a better understanding of medical professionalism to enhance the assessment and ultimately, the teaching of medical professionalism in an urban multi-ethnic Asian city.
\end{abstract}

Keywords: Professionalism, Singapore, Qualitative, Assessment

\section{Background}

In recent years, medical professionalism has been increasingly emphasized in medical undergraduate and post-graduate curricula [1-4]. Previously 'good medical practice' has been defined more broadly in terms of roles such as 'professional' and 'manager', with various medical councils, academic and professional bodies having produced clear documentations on these roles [5-7]. In 2002, a set of professional attributes was defined in the Physician's charter on Medical Professionalism, defining a professional doctor as having professional competence, being honest with patients, maintaining patients'

\footnotetext{
* Correspondence: warren.fong.w.s@singhealth.com.sg

${ }^{1}$ Department of Rheumatology and Immunology, Singapore General Hospital, Singapore, Singapore

2Duke-NUS Medical School, Singapore, Singapore

Full list of author information is available at the end of the article
}

confidentiality, maintaining appropriate relationships with patients, being able to improve quality of care, ensuring just distribution of resources, possessing scientific knowledge, being able to manage conflicts of interest and possessing professional responsibilities [1]. The charter is overseen by leaders in the American Board of Internal Medicine (ABIM) Foundation, the American College of Physicians-American Society of Internal Medicine (ACP-ASIM) Foundation, and the European Federation of Internal Medicine [1]. To date, the charter has been endorsed by 108 national and international organizations [8]. Medical professionalism has been shown to affect doctors' relationships with their patients, quality of care, and ultimately health and illness outcomes [9]. For residents in training, unprofessional behaviour during their training resulted in increased risk of

(c) The Author(s). 2020 Open Access This article is distributed under the terms of the Creative Commons Attribution 4.0 International License (http://creativecommons.org/licenses/by/4.0/), which permits unrestricted use, distribution, and reproduction in any medium, provided you give appropriate credit to the original author(s) and the source, provide a link to the Creative Commons license, and indicate if changes were made. The Creative Commons Public Domain Dedication waiver (http://creativecommons.org/publicdomain/zero/1.0/) applies to the data made available in this article, unless otherwise stated. 
disciplinary action later on in their careers as physicians [10]. Fortunately, medical professionalism can be nurtured [11]. Thus, assessment of medical professionalism in daily practice of trainees becomes increasingly important as part of their development of their professional identity, with the aim of being able to provide timely feedback and early remediation [12].

There have been studies attempting to define the domains of professionalism using qualitative methods [13-16]. Wagner et al. has identified knowledge/technical skills, patient relationship and character virtues as main themes of professionalism [13], while tha et al. has identified compliance to values, patient access, doctor-patient relationship, demeanour, professional management, personal awareness and motivation as the themes of professionalism [14]. However, none of these studies were performed in the context of Asian medical education. As highlighted by the cultural value theory and cultural dimension theory, cultural differences exist between countries $[17,18]$. Previous study has shown that the conceptualization of professionalism is influenced by culture [19-21]. For example, altruism was found to be an essential element of medical professionalism for Asia and North America, but not Europe [21]. In addition, the constituents of medical professionalism in China was influenced by its longstanding Confucian traditions [22].

We aim to describe the views and experiences of both patients and faculty on medical professionalism in an urban multi-ethnic Asian city. In doing so, we hope to be able to improve our understanding of medical professionalism in Asia and to develop culturally-adapted tools to measure and improve medical professionalism.

\section{Methods}

\section{Study design}

We used semi-structured interviews to elicit participants' views and experiences of medical professionalism from December 2017 to October 2018. We anchored our methodology according to the Consolidated Criteria for Reporting Qualitative Research (COREQ) checklist (Supplementary data) [23]. The following purposive sampling technique was performed to provide a range of views on medical professionalism: recipients of healthcare services were selected based on age, gender, ethnicity, education and socio-economic status while medical educators responsible for medical training and assessment were selected according to age, gender, ethnicity and disciplines for faculty in SingHealth residency. SingHealth is Singapore's largest group of public healthcare institutions, consisting of four public hospitals, five national specialty centres and a network of community hospitals and polyclinics [24]. There are over 1600 faculty and more than 900 residents in training, with yearly outpatient attendances of about 4 million. As participants accepted the invitations and scheduled interviews, we iteratively adjusted our subsequent invitations to ensure a reasonably balanced representation of recipients of healthcare services and medical educators. Some participants might be colleagues or patients of the researchers (WF, JT, YYL and SCN). However, these researchers were not involved in the recruitment and interview processes to minimise potential influence on the participants.

\section{Focus group discussions (FGDs) with patients}

We invited patients who had received outpatient and/or inpatient care in any healthcare facilities to participate in FGDs through telephone and email after referral from attending doctors in different disciplines. One patient refused to participate due to discomfort with the interview. We conducted FGDs with patients in English or Mandarin in a quiet room. Each FGD lasted approximately 75 to $90 \mathrm{~min}$. All FGDs were facilitated by a moderator, in the presence of another study team member who served as an observer and a note taker. The moderators had no prior relationship with the participants prior to study commencement. During session introductions, interviewers shared only their name, job title, and role in the project with study participants.

Moderators used a standardized guide (see supplementary materials) to identify issues related to medical professionalism that were important to patients. Our systematic review had earlier indicated that P-MEX was one of the promising tools to assess medical professionalism [25]. Therefore, we developed our topic guide based on the domains and items of the P-MEX, with an intent to adapt this tool for use in Singapore. The original P-MEX consists of 4 domains (Doctor-patient relationship skills, Reflective skills, Time management and Inter-professional relationship skills) and 21 subdomains [26]. We started with questions that were open-ended, with some prompts to ensure consistency and coverage of topics across groups. The interview guide was pilot-tested. We divided the FGD into 2 parts. First, patients were asked to list the traits they considered important for professionalism in all doctors. Patients were then asked to discuss these traits in the group discussion. Next, patients were asked to discuss the 10 items from the Doctor-patient relationship and Reflective skills domains of P-MEX in terms of assessing demeanours of a professional doctor in the local cultural and healthcare context. The patients were also invited to list any missing item which may be important for the assessment of a professional doctor. Focus groups were conducted until data saturation was reached. 
In-depth interviews (IDIs) with medical faculty

As it was difficult to coordinate the schedules among faculty members, we conducted semi-structured IDIs with faculty members in English in a quiet room. Each IDI lasted approximately $30-45 \mathrm{~min}$. All IDIs were facilitated by the same moderator as the FGD. The standardized guide was similar to the one used for FGD, but for the second part, faculty members were asked if there were any items that they felt were not relevant, and to choose up to five least relevant items in assessing medical professionalism if applicable. The faculty were also invited to list any missing item which may be important for the assessment of a professional doctor. Interviews were conducted until data saturation was achieved.

\section{Data analysis}

FGDs and IDIs were voice-recorded and transcribed verbatim. Thematic data analysis was conducted by two independent coders (YHK and JKP), who were both trained in qualitative research, to ensure inter-coder reliability. The method of analysis chosen for this study was a hybrid approach of qualitative methods of thematic analysis, and it incorporated both the inductive approach based on the grounded theory and the deductive approach using a priori template of codes [27, 28]. Elements of grounded theory was adopted in the analysis of the data, allowing new codes and categories outside of the P-MEX to emerge during the analysis of each transcript, encouraging the development of a conceptual framework from the input of participants. For example, when provision of clear and honest information was discussed extensively by participants, it was selected as one open coding category, positioning it as a central category of the indicators. Provided clear and honest information was subsequently recoded into communicated effectively with patient (axial coding) when similar categories emerged from the data such as communicated empathetically. We used NVivo 11 software to facilitate the data analysis process.

The four domains of medical professionalism from the P-MEX (Doctor-patient relationship skills, Reflective skills, Time management and Inter-professional relationship skills) were used as a priori coding template to support the analysis [26]. The initial cycle of coding was done by deductive approach using a priori template of codes, and the second cycle of coding was done to identify any new domains or sub-domains using inductive approach. The primary researchers (YHK and JKP) discussed each stage of the analysis with the research team (WF, SY, YYL, SCN). Discrepancies in interpretation of materials were resolved through an iterative discussion amongst research team members until a list of codes that could be consistently applied was compiled.
In order to maintain the methodological rigour of qualitative research, the following strategies were systematically applied to our study based on the Lincoln and Guba's four criteria [29]: credibility was achieved through pilot testing of the topic guides and collection and inclusion of field notes for data analysis. We have also reported preliminary findings at several scientific meetings to gain insights and views from relevant parties; dependability was achieved by a detailed track record of the data collection process and assessment of coding accuracy and inter-coder agreement among the research team throughout the analysis process; confirmability was achieved through data triangulation (i.e. interviews with both patients and faculty); and transferability was achieved through purposive sampling to ensure that the selected participants were representative of the views of patients and faculty. We also employed iterative interpretations of findings until no new codes emerged from the dataset and all variations in key concepts were identified.

\section{Ethics}

The SingHealth Centralized Institutional Review Board approved this study (Ref No: 2016/3009). We obtained informed consent, which conformed to the principle outlined in the 1964 Declaration of Helsinki, from all patients and faculty before the interview.

\section{Results}

A total of 31 patients ( $58.1 \%$ male, median age 32 years old, age range from 22 to 75 years old) participated in 6 FGDs (4 conducted in English and 2 conducted in Mandarin) while a total of 26 faculty members $(42.3 \%$ male, median age 42 years old, age range from 26 to 76 years old) participated in the IDIs (all conducted in English). All patients had visited healthcare facilities within the past year (Table 1). Based on the data from 2018 from the Department of Statistics Singapore [30], the majority of the residents are Chinese (74\%), followed by Malays (13\%) and Indians (9\%). The percentage of residents with post-secondary education is $55.8 \%$. The median age of the population is 40.8 years. One-third of the faculty members came from medical disciplines, the rest spread across a wide spectrum of disciplines (anesthesiology, surgical, diagnostic radiology, nuclear medicine and pathology, emergency medicine, paediatrics, surgical and allied health). The FGDs ranged in size from 4 to 6 participants. Data saturation occurred after 18 IDIs and 5 FGDs, with no new themes emerging. The socio-demographic characteristics of the participants in the FGD and IDI are summarized in Tables 1 and 2.

\section{Framework of medical professionalism}

The framework for medical professionalism for this study is shown in Fig. 1. Using the a priori framework 
Table 1 Demographics profile of patients who participated in focus group discussions $(n=31)$

\begin{tabular}{|c|c|}
\hline Characteristics & Number (\%) \\
\hline \multicolumn{2}{|l|}{ Age } \\
\hline $21-29$ & $12(38.7)$ \\
\hline $30-39$ & $6(19.4)$ \\
\hline $40-49$ & $1(3.2)$ \\
\hline $50-59$ & $6(19.4)$ \\
\hline $60-69$ & $4(12.9)$ \\
\hline $70-79$ & $2(6.5)$ \\
\hline \multicolumn{2}{|l|}{ Gender } \\
\hline Male & $18(58.1)$ \\
\hline Female & $13(41.9)$ \\
\hline \multicolumn{2}{|l|}{ Ethnicity } \\
\hline Chinese & $27(87.1)$ \\
\hline Malay & $2(6.5)$ \\
\hline Indian & $2(6.5)$ \\
\hline \multicolumn{2}{|l|}{ Highest education attained } \\
\hline Secondary and below & $5(16.1)$ \\
\hline Post- secondary & $26(83.9)$ \\
\hline \multicolumn{2}{|l|}{ Marital status } \\
\hline Single & $18(58.1)$ \\
\hline Married & $13(41.9)$ \\
\hline \multicolumn{2}{|l|}{ Employment status } \\
\hline Employed & $18(58.1)$ \\
\hline Unemployed & $13(41.9)$ \\
\hline \multicolumn{2}{|l|}{ Housing } \\
\hline Public housing & $18(58.0)$ \\
\hline Private housing & $11(35.5)$ \\
\hline Hostel & $2(6.5)$ \\
\hline \multicolumn{2}{|c|}{ Healthcare facility visited in the past 1 year } \\
\hline Polyclinic & $18(58.1)$ \\
\hline General practitioner & $20(64.5)$ \\
\hline Hospital admission & $6(19.4)$ \\
\hline Specialist outpatient clinic & $13(41.9)$ \\
\hline
\end{tabular}

for medical professionalism of P-MEX, 4 domains were derived (Doctor-patient relationship skills, Reflective skills, Time management and Inter-professional relationship skills). There were 21 codes which were mapped against the P-MEX. Three new codes emerged from the data. These codes were subsequently merged into 23 sub-domains and 4 domains (Table 3).

\section{Doctor-patient relationship}

Our data mapped against all 7 original sub-domains in doctor-patient relationship, namely (1) listened actively to patient, (2) showed interest in patient as a person, (3)
Table 2 Demographics profile of faculty members who participated in interviews $(n=26)$

\begin{tabular}{|c|c|}
\hline Characteristics & Number (\%) \\
\hline \multicolumn{2}{|l|}{ Age } \\
\hline $21-29$ & $2(7.7)$ \\
\hline $30-39$ & $8(30.8)$ \\
\hline $40-49$ & $11(42.3)$ \\
\hline $50-59$ & $3(11.5)$ \\
\hline $60-69$ & $0(0)$ \\
\hline $70-79$ & $2(7.7)$ \\
\hline \multicolumn{2}{|l|}{ Gender } \\
\hline Male & $11(42.3)$ \\
\hline Female & $15(57.7)$ \\
\hline \multicolumn{2}{|l|}{ Years as faculty } \\
\hline $2-10$ & $13(50.0)$ \\
\hline$>10$ & $13(50.0)$ \\
\hline \multicolumn{2}{|l|}{ Ethnicity } \\
\hline Chinese & $17(65.4)$ \\
\hline Malay & $1(3.8)$ \\
\hline Indian & $8(30.8)$ \\
\hline \multicolumn{2}{|l|}{ Disciplines } \\
\hline Medical disciplines ${ }^{\text {a }}$ & $10(38.5)$ \\
\hline Anesthesiology & $2(7.7)$ \\
\hline Diagnostic radiology, Nuclear Medicine and Pathology & $2(7.7)$ \\
\hline Emergency medicine & $1(3.8)$ \\
\hline Paediatrics & $2(7.7)$ \\
\hline Surgical disciplines ${ }^{\text {b }}$ & $3(11.5)$ \\
\hline Allied Health ${ }^{c}$ & $6(23.1)$ \\
\hline
\end{tabular}

a includes respiratory medicine, dermatology, neurology, nephrology, internal medicine, infectious disease

$\mathrm{b}$ includes general surgery, obstetrics and gynaecology

c includes pharmacy, physiotherapy, medical social service, podiatry, nursing, and occupational therapy

recognized and met patient needs, (4) extended his or herself to meet patient needs, (5) ensured continuity of patient care, (6) advocated on behalf of a patient, (7) maintained appropriate boundaries. However, the subdomain "extended his or herself to meet patient needs" was deemed to be less relevant by both faculty members and patients. They felt that extending oneself to meet the needs of the patient may be impractical, as some needs may be beyond the doctor's ability to fulfil. The faculty and patients felt that it would be sufficient if a doctor could fulfil the requirements of other subdomains, as shown in the following quote "I think what is needed here is just the doctor to actively listen. I think that's enough, because to extend the extra effort, the doctor may not have enough time." (Patient, 22 years old, Male, Chinese). 


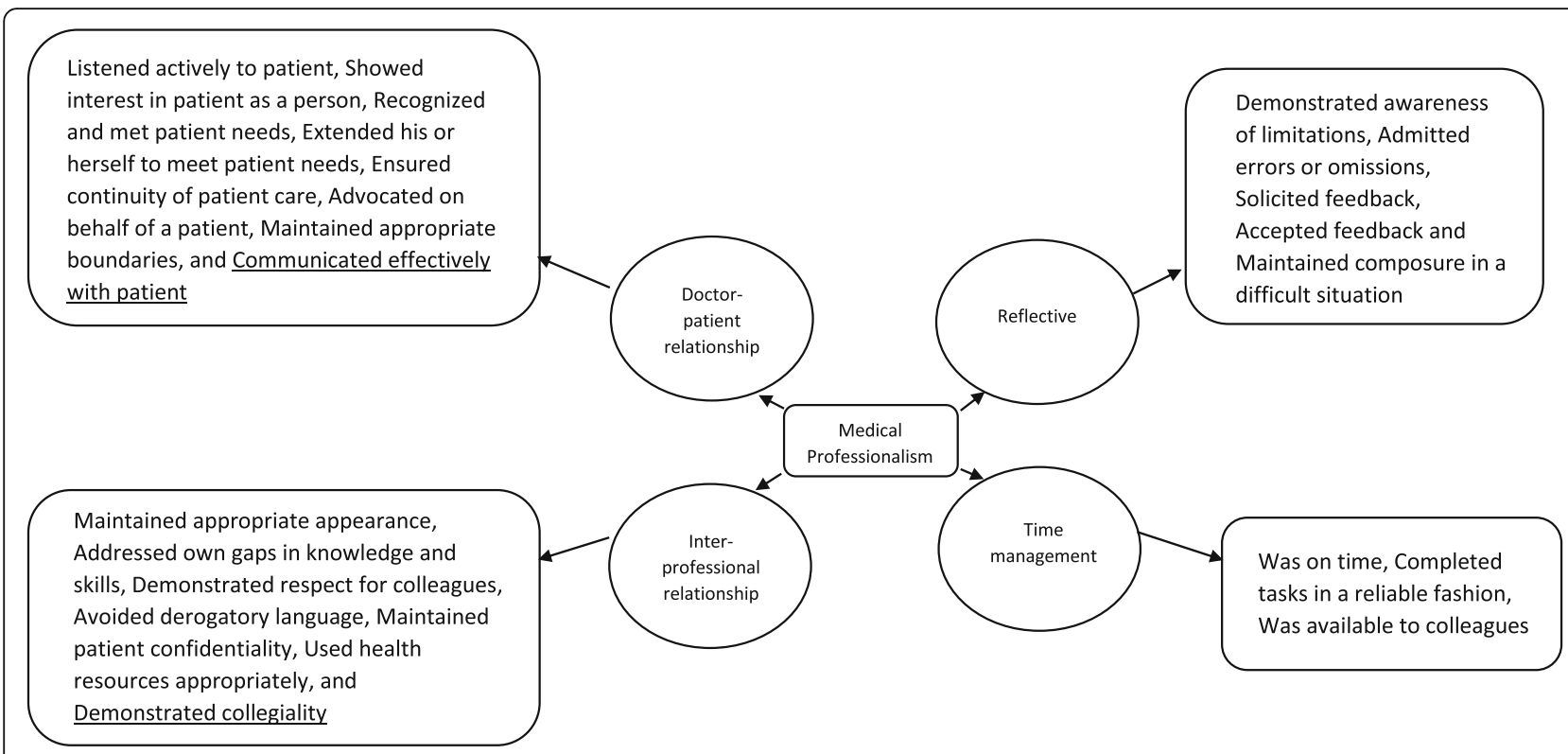

Fig. 1 Domains and subdomains of medical professionalism. Underlined sub-domains are the new sub-domains derived from this study

We also found a new sub-domain, "communicated effectively with patient". It was repeatedly highlighted by patients that it was important for a professional doctor to take time to explain the disease, treatment options, risks and benefits, and ensure that patients understood. Faculty also stressed the importance of communication skills for a professional doctor.

\section{Reflective skills}

Our data mapped against all 5 original sub-domains in reflective skills, namely (1) demonstrated awareness of limitations, (2) admitted errors or omissions, (3) solicited feedback, (4) accepted feedback and (5) maintained composure in a difficult situation.

The sub-domain "solicited feedback" was deemed to be less relevant by faculty members. Faculty members commented that feedback will be given to trainees, therefore it is not necessary for the trainees to seek feedback actively. Patients also felt uncomfortable if doctors solicited feedback from them, as evident from the following quote: "It makes me completely lose confidence if the doctor ask me how he/she is doing." (Patient, 75 years old, Female, Chinese).

\section{Time management}

Our data mapped against all 3 original sub-domains in time management, namely namely (1) was on time, (2) completed tasks in a reliable fashion, (3) was available to colleagues.

The faculty clarified that while respect for other people's time was important and doctors should strive to be punctual, there are circumstances where doctors can be delayed, for example, medical emergencies and complicated patients who requires a longer time to manage.

\section{Inter-professional relationship}

Our data mapped against all 6 original sub-domains in inter-professional relationship, namely (1) maintained appropriate appearance, (2) addressed own gaps in knowledge and skills, (3) demonstrated respect for colleagues, (4) avoided derogatory language, (5) maintained patient confidentiality, (6) used health resources appropriately. We also found a new sub-domain, "demonstrated collegiality". Faculty stressed the importance of teamwork in medicine, where it was important to respect the contribution of each healthcare worker in the care of the patient, and cooperating with one another to ensure the best outcome in the care of the patient. This is evident in the following quote, "Medical care for the patient is a teamwork, because doctors can make all the orders, but you need the nurses to carry out the orders and of course you need a lot of help from allied health professionals, like physiotherapists, occupational therapists, dieticians, speech therapists." (Faculty, 32 years old, Female, Chinese).

Faculty members did not completely agree as to what is "appropriate use of health resources". Some faculty members felt that overutilization or underutilization of health resources such as hospital beds, expensive drugs and scans, may be due to other reasons such patient's financial resources and personal convictions. For example, faculty mentioned that patients who had the financial resources might request for more investigations than needed. 


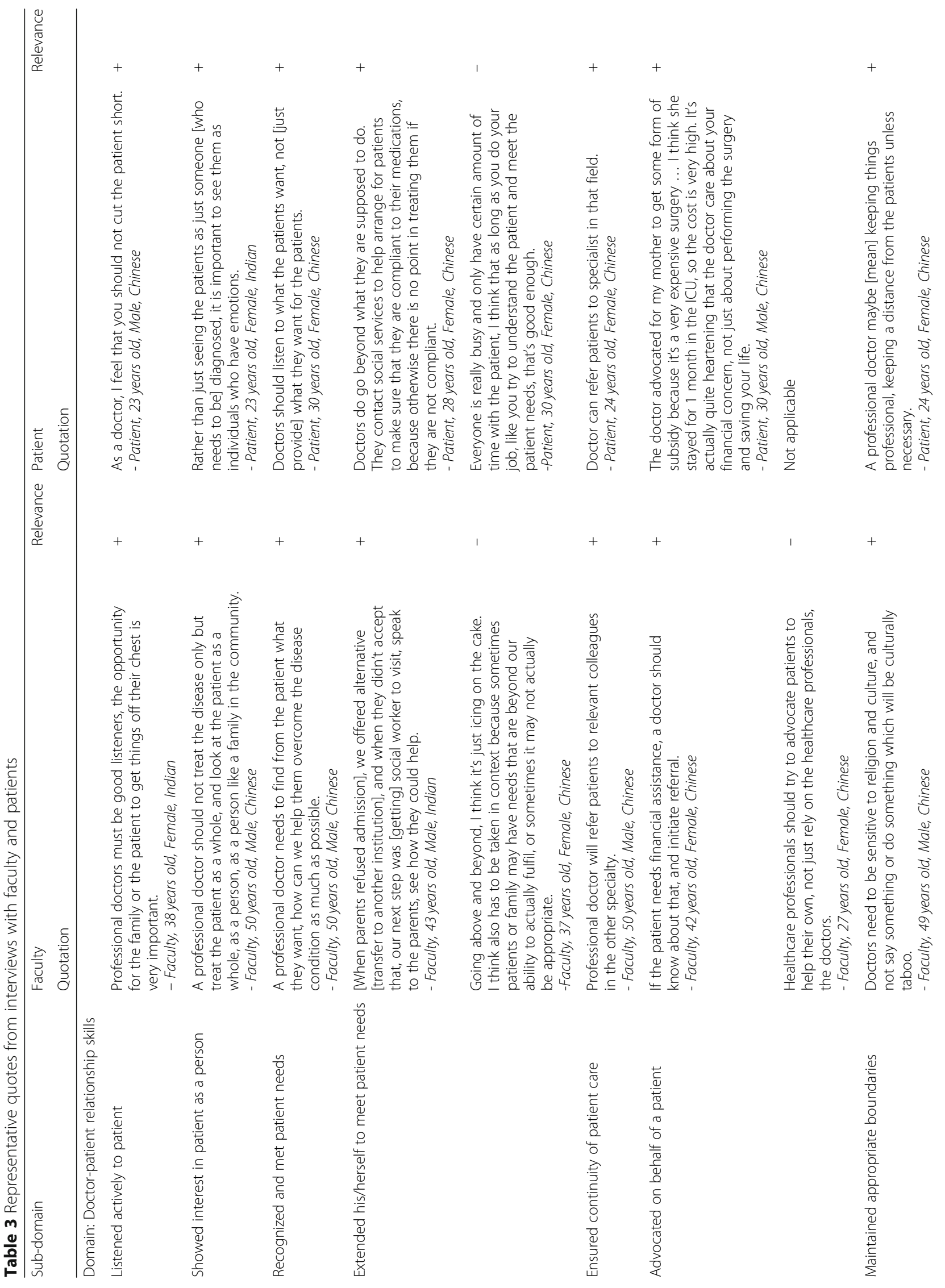




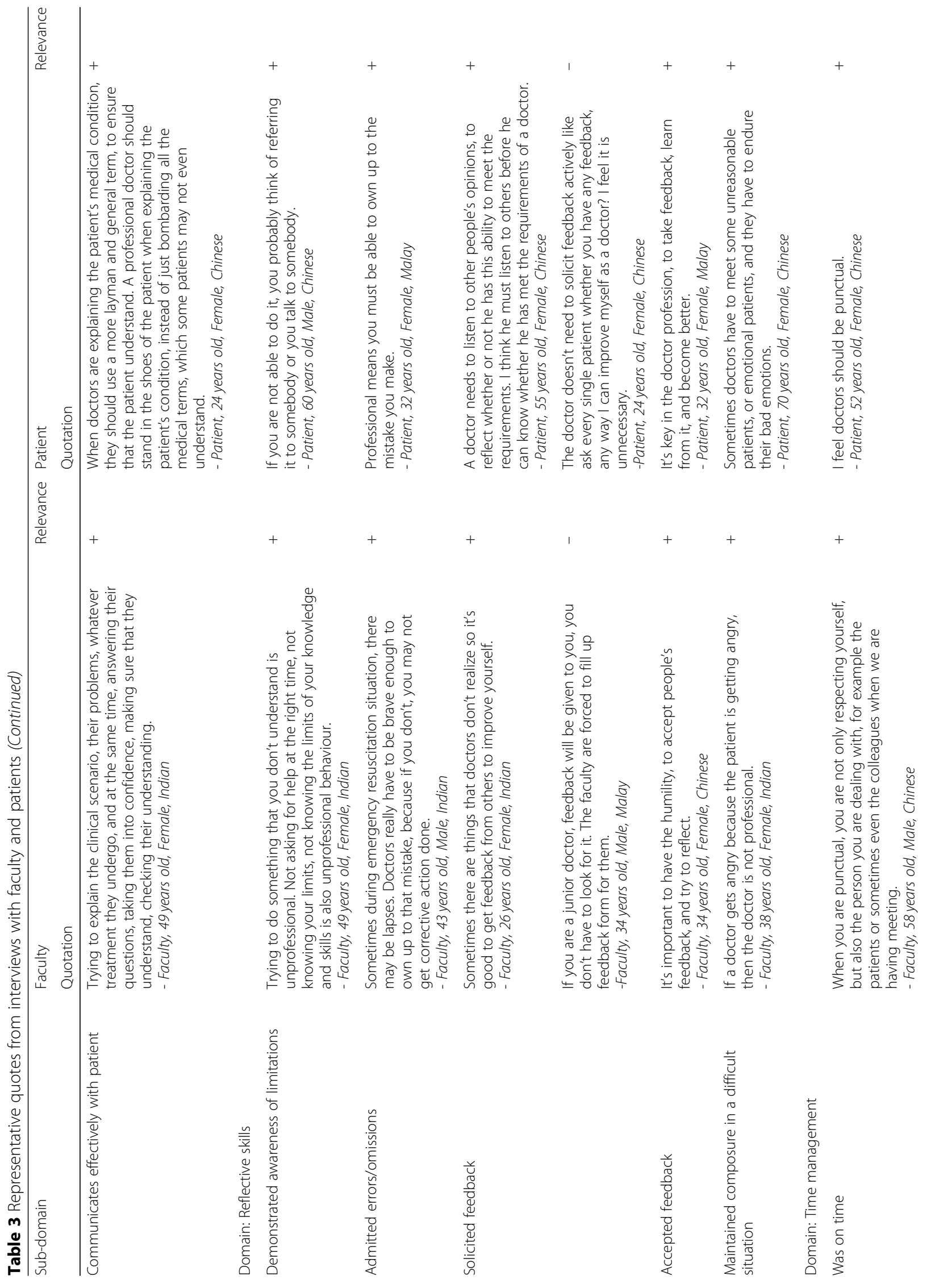




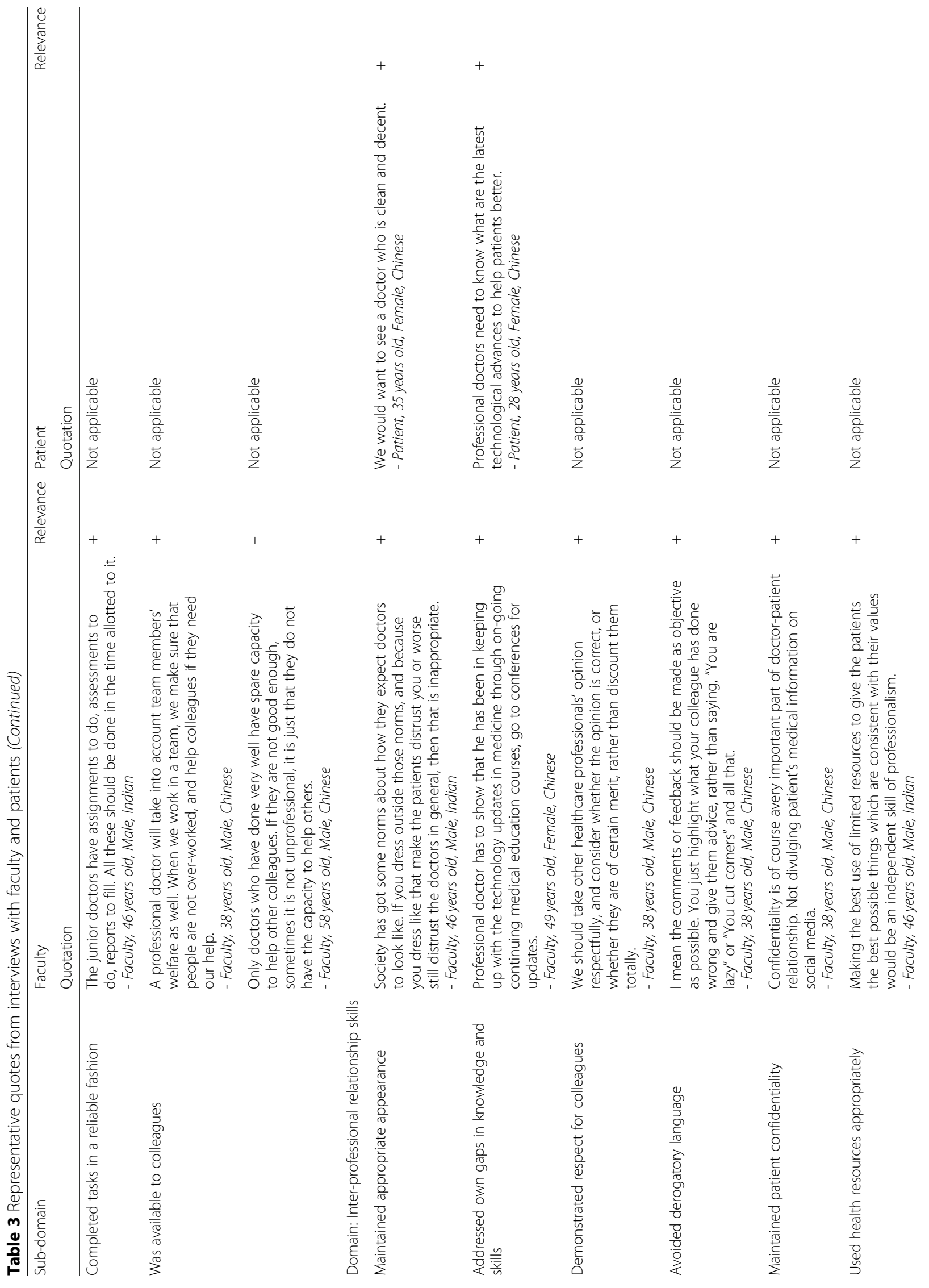




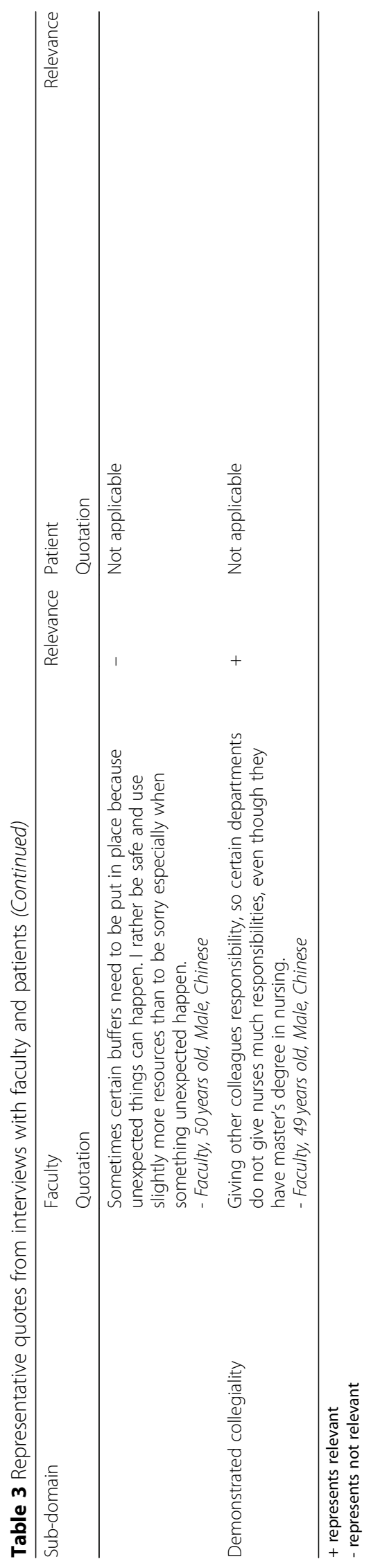


Least relevant sub-domains as deemed by faculty

The top three least relevant sub-domains as chosen by the faculty were "solicited feedback" $(n=17)$, "extended his or herself to meet patient needs" $(n=16)$, "used health resources appropriately" $(n=15)$. As mentioned previously, the faculty members commented that feedback will be given to trainees routinely, therefore it is not necessary for the trainees to seek feedback actively. The faculty also felt that extending oneself to meet the needs of the patient may be impractical. The faculty also commented that the inappropriate use of health resources by trainees may "not be because they were unprofessional but due to inexperience" (Faculty, 32 years old, Female, Chinese).

\section{Discussion}

The results from our current study supported the four domains of medical professionalism derived from the PMEX [26], namely Doctor-patient relationship skills, Reflective skills, Time management and Inter-professional relationship skills. In addition, 2 new sub-domains "communicated effectively with patient" and "demonstrated collegiality" under the domains of Doctor-patient relationship skills and Inter-professional relationship were identified respectively. The least relevant sub-domains as chosen by the faculty were "solicited feedback", "extended his or herself to meet patient needs", "used health resources appropriately".

The four domains of medical professionalism identified in this study also are similar to the components of medical professionalism as highlighted by the General Medical Council, namely "Behave according to ethical and legal principles", "Reflect, learn and teach others", "Learn and work effectively within a multi-professional team", "Protect patients and improve care" [7]. Previous studies have identified knowledge/technical skills, patient relationship, character virtues, compliance to values, patient access, doctor-patient relationship, demeanour, professional management, personal awareness and motivation as main themes of professionalism [13, 14]. These themes of professionalism can be found in domains and sub-domains of professionalism in this study.

Our study has also identified new sub-domains not present in the original P-MEX. Under the Doctorpatient relationship skills domain, the new sub-domain is "communicated effectively with patient". The emergence of the new sub-domain "communicated effectively with patient" illustrates the shift in medical care towards one with emphasis on patient autonomy [31]. Traditionally, doctor-patient relationships were shaped by paternalism where doctors had a high degree of control over the patients, and this was more apparent in Asian context [32]. However, the communication model is moving towards more egalitarian partnership, with greater awareness of medical consumerism [33]. The importance of communication skills in medical professionalism has also been highlighted in other studies [19, 21]. Under the Interprofessional relationship skills domain, we identified a new sub-domain "demonstrated collegiality". According to Hofstede's cultural dimension theory, Asians tend to be more collectivist compared to Western populations [18], and this was highlighted in the new sub-domain "demonstrated collegiality", which demonstrates the faculty's perception of the increasing importance of team-based care and collaboration between various healthcare professionals, whose opinions should be respected [34, 35]. The importance of collegiality was also highlighted in the study by Chandratilake et al. who showed that working with one's colleagues towards common goals was deemed to be important in European and North American countries [21].

The strengths of this study include purposive sampling to ensure that a broad range of views about medical professionalism elicited from both patients and faculty. The patients recruited for this study mirrored the ethnicity distribution of the general population in Singapore [36]. In addition, we included patients of different age groups and patients attending primary care as well as tertiary care institutions in the FGDs. We also ensured that faculty members across different disciplines were included in the IDIs. To the best of our knowledge, this is the first qualitative study performed in Asia to explore medical professionalism.

Limitations of this study include limited generalizability of findings to other Asian countries as this study was done in a single Asian country, and perception of medical professionalism is affected by different cultural context. However, since there few studies exploring medical professionalism in Asia, this study provides a basis for conducting future research on medical professionalism, especially in Asia. Secondly, patients who were purely Malay and Tamil speakers were not included. However, the impact of this on the results is likely to be small, given that pure Malay and Tamil speakers only formed 1.2 and $0.29 \%$ of the resident population in 2015 [37]. In addition, the coding frame was based on the domains of medical professionalism from P-MEX. Therefore, we may have missed certain attributes of medical professionalism that were not included in the P-MEX. However, the qualitative nature of our study provided a nuanced understanding of medical professionalism within the context of an Asian healthcare setting, identifying two new sub-domains. The percentage of residents with postsecondary education was $84 \%$ in our study, which was higher than that of the general population (55.8\%). However, this is not likely to have an impact as difference in perception of professionalism for patients with different education level was not observed for our study and other study as well [38]. 


\section{Conclusion}

In conclusion, we found that Doctor-patient relationship skills, Reflective skills, Time management and Interprofessional relationship skills are relevant to both faculty members and patients as domains of medical professionalism. New sub-domains such as "communicating effectively with the patient" and "demonstrated collegiality" were also found to be important to medical professionalism in Singapore. Future research in Asian countries may consider including these sub-domains for assessment of medical professionalism.

\section{Abbreviations}

ABIM: American Board of Internal Medicine; ACP-ASIM: American College of Physicians-American Society of Internal Medicine; COREQ: Consolidated Criteria for Reporting Qualitative Research; FGD: Focus group discussion; IDI: In-depth interviews; P-MEX: Professionalism Mini Evaluation Exercise

\section{Acknowledgements}

The authors thank all the participants for their time and contribution to the study. The authors also acknowledge Joanna Yeo and Emily Luo Zhifei for their contributions as moderators for the interviews and focus group discussions.

\section{Authors' contributions}

WF, YHK, SWY, YYL, JT and SCN conceptualized the study design, and drafted the qualitative interview guide book. YHK and JKP coded the transcript verbatim. WF, YHK, SWY and JKP wrote the initial manuscript draft. All authors critically reviewed and approved the final manuscript. SCN, YYL, JT and WF are consultant physicians and medical educators at Singapore General Hospital. SWY, YYL, JT and YHK are qualitative researchers with extensive experience. JKP is research coordinator at Singapore General Hospital.

\section{Funding}

This research was supported by SingHealth Duke-NUS Medicine Academic Clinical Programme Education Support Programme Grant (Ref No: 03/ FY2017/P2/03-A47). Funder was not involved in the design, delivery or submission of the research.

\section{Availability of data and materials}

The datasets used and/or analysed during the current study are available from the corresponding author on reasonable request.

\section{Ethics approval and consent to participate}

The SingHealth Centralized Institutional Review Board approved this study (Ref No: 2016/3009). We obtained informed consent, which conformed to the principle outlined in the 1964 Declaration of Helsinki, from all patients and faculty before the interview.

\section{Consent for publication}

Not applicable.

\section{Competing interests}

The authors declare that they have no competing interests.

\section{Author details}

'Department of Rheumatology and Immunology, Singapore General Hospital, Singapore, Singapore. ${ }^{2}$ Duke-NUS Medical School, Singapore, Singapore. ${ }^{3}$ NUS Yong Loo Lin School of Medicine, National University of Singapore, Singapore, Singapore. ${ }^{4}$ Sing Health Rheumatology Senior Residency Programme, 20 College Road, Singapore 169856, Singapore. ${ }^{5}$ Program in Health Services and Systems Research, Duke-NUS Medical School, Singapore, Singapore.
Received: 26 July 2019 Accepted: 22 January 2020

Published online: 30 January 2020

\section{References}

1. Medicine AFABol. Medical professionalism in the new millennium: a physician charter. Ann Intern Med. 2002;136:243.

2. Swick HM, Szenas $P$, Danoff D, Whitcomb ME. Teaching professionalism in undergraduate medical education. JAMA. 1999;282:830-2.

3. Cruess RL, Cruess SR. Teaching professionalism: general principles. Med Teach. 2006;28:205-8.

4. Tweedie Jude JH, Dacre DJ. Advancing medical professionalism London Royal College of Physicians; 2018.

5. Blank L, Kimball H, McDonald W, Merino J. Medical professionalism in the new millennium: a physician charter 15 months later. Ann Intern Med. 2003;138:839-41.

6. Frank JR. The CanMEDS 2005 physician competency framework. Better standards. Better physicians. Better care. The Royal College of Physicians and Surgeons of Canada: Ottawa; 2005.

7. Tomorrow's Doctors. General medical council 2009.

8. ABIM Foundation. Endorsements of The Charter 2019 [https:// abimfoundation.org/what-we-do/physician-charter/endorsements-of-thecharter].

9. Blasi ZD, Harkness E, Ernst E, Georgiou A, Kleijnen J. Influence of context effects on health outcomes: a systematic review. Lancet. 2001:357:757-62.

10. Papadakis MA, Hodgson CS, Teherani A, Kohatsu ND. Unprofessional behavior in medical school is associated with subsequent disciplinary action by a state medical board. Acad Med. 2004;79:244-9.

11. O'Sullivan $H$, van Mook W, Fewtrell $R$, Wass V. Integrating professionalism into the curriculum: AMEE guide no. 61. Med Teach. 2012;34:e64-77.

12. Eisner EW. The educational imagination: on the design and evaluation of school programs. 3rd ed. New Jersey: Prentice Hall; 1979.

13. Wagner P, Hendrich J, Moseley G, Hudson V. Defining medical professionalism: a qualitative study. Med Educ. 2007:41:288-94.

14. Jha V, Bekker $H$, Duffy $S$, Roberts T. Perceptions of professionalism in medicine: a qualitative study. Med Educ. 2006:40:1027-36.

15. Cuesta-Briand B, Auret K, Johnson P, Playford D. A world of difference': a qualitative study of medical students' views on professionalism and the 'good doctor. BMC Med Educ. 2014;14:77.

16. Finn G, Garner J, Sawdon M. 'You're judged all the time!'students' views on professionalism: a multicentre study. Med Educ. 2010:44:814-25.

17. Schwartz SH. A theory of cultural values and some implications for work. Appl Psychol. 1999:48:23-47.

18. Hofstede G. National cultures in four dimensions: a research-based theory of cultural differences among nations. Int Stud Manage Organ. 1983:13:46-74.

19. Van De Camp K, Vernooij-Dassen MJ, Grol RP, Bottema BJ. How to conceptualize professionalism: a qualitative study. Med Teach. 2004:26:696-702.

20. Cruess SR, Cruess RL, Steinert Y. Teaching professionalism across cultural and national borders: lessons learned from an AMEE workshop. Med Teach. 2010;32:371-4.

21. Chandratilake M, McAleer S, Gibson J. Cultural similarities and differences in medical professionalism: a multi-region study. Med Educ. 2012;46:257-66.

22. Jin P. The physician charter on medical professionalism from the Chinese perspective: a comparative analysis. J Med Ethics. 2015:41:511-4.

23. Tong A, Sainsbury P, Craig J. Consolidated criteria for reporting qualitative research (COREQ): a 32-item checklist for interviews and focus groups. Int J Qual Health Care. 2007;19:349-57.

24. Ong B-H, Gonzalez-Rivas D. The inaugural SingHealth uniportal videoassisted thoracoscopic surgery masterclass in Singapore. J Vis Surg. 2018;4.

25. Kwan $Y H$, Png K, Phang JK, Leung YY, Goh H, Seah Y, Thumboo J, Ng APSC, Fong W, Lie D. A systematic review of the quality and utility of observerbased instruments for assessing medical professionalism. J grad Med Educ. 2018;10:629-38

26. Cruess R, Mcllroy JH, Cruess S, Ginsburg S, Steinert Y. The professionalism minievaluation exercise: a preliminary investigation. Acad Med. 2006:81:574-8.

27. Fereday J, Muir-Cochrane E. Demonstrating rigor using thematic analysis: a hybrid approach of inductive and deductive coding and theme development. Int J Qual Methods. 2006;5:80-92.

28. Glaser BG: Strauss. AL (1967). The discovery of grounded theory: Strategies for qualitative research.

29. Lincoln Y, Guba E. Naturalistic inquiry. 1st ed. Newbury Park: Sage Publications Inc; 1985. 
30. Population Trends 2018 [https://www.singstat.gov.sg/-/media/files/ publications/population/population2018.pdf].

31. Quill TE, Brody H. Physician recommendations and patient autonomy: finding a balance between physician power and patient choice. Ann Intern Med. 1996;125:763-9.

32. Claramita M, Nugraheni MDF, van Dalen J, van der Vleuten C. Doctorpatient communication in Southeast Asia: a different culture? Adv Health Sci Educ Theory Pract. 2013;18:15-31.

33. Coulter A. Paternalism or partnership? Patients have grown up-and there's no going back, vol. 319; 1999. p. 719-20.

34. Chua AZ, Lo DY, Ho WH, Koh YQ, Lim DS, Tam JK, Liaw SY, Koh GC. The effectiveness of a shared conference experience in improving undergraduate medical and nursing students' attitudes towards interprofessional education in an Asian country: a before and after study. BMC Med Ed. 2015:15:233.

35. Zheng RM, Sim YF, Koh GC-H. Attitudes towards interprofessional collaboration among primary care physicians and nurses in Singapore. J Interpro Care. 2016; 30:505-11.

36. Census of Population. Statistical Release. 2010:1 https://www.singstat.gov.sg/-/ media/files/publications/cop2010/census_2010_release1/cop2010sr1.pdf.

37. Statistics Do. General household survey 2015: Ministry of Trade \& Industry, Republic of Singapore; 2016.

38. Taibah SM. Dental professionalism and influencing factors: patients' perception. Patient preference adherence. 2018;12:1649.

\section{Publisher's Note}

Springer Nature remains neutral with regard to jurisdictional claims in published maps and institutional affiliations.

Ready to submit your research? Choose BMC and benefit from:

- fast, convenient online submission

- thorough peer review by experienced researchers in your field

- rapid publication on acceptance

- support for research data, including large and complex data types

- gold Open Access which fosters wider collaboration and increased citations

- maximum visibility for your research: over $100 \mathrm{M}$ website views per year

At $\mathrm{BMC}$, research is always in progress.

Learn more biomedcentral.com/submissions 